\title{
Need for Contraceptive Services Among Women of Reproductive Age - 45 Jurisdictions, United States, 2017-2019
}

Lauren B. Zapata, $\mathrm{PhD}^{1}$; Karen Pazol, $\mathrm{PhD}^{2}$; Kathryn M. Curtis, $\mathrm{PhD}^{1}$; Debra J. Kane, $\mathrm{PhD}^{3}$; Tara C. Jatlaoui, $\mathrm{MD}^{4}$; Suzanne G. Folger, PhD ${ }^{1}$; Ekwutosi M. Okoroh, $\mathrm{MD}^{1}$; Shanna Cox, $\mathrm{MSPH}^{1}$; Maura K. Whiteman, $\mathrm{PhD}^{1}$

Ensuring access to contraceptive services is an important strategy for preventing unintended pregnancies, which account for nearly one half of all U.S. pregnancies (1) and are associated with adverse maternal and infant health outcomes (2). Equitable, person-centered contraceptive access is also important to ensure reproductive autonomy (3). Behavioral Risk Factor Surveillance System (BRFSS) data collected during 2017-2019 were used to estimate the proportion of women aged 18-49 years who were at risk for unintended pregnancy* and had ongoing or potential need for contraceptive services. ${ }^{\dagger}$ During 2017-2019, in the 45 jurisdictions ${ }^{\S}$ from which data were collected, $76.2 \%$ of women aged 18-49 years were considered to be at risk for unintended pregnancy, ranging from $67.0 \%$ (Alaska) to $84.6 \%$ (Georgia); $60.7 \%$ of women had ongoing or potential need for contraceptive services, ranging from $45.3 \%$ (Puerto Rico) to $73.7 \%$ (New York). For all jurisdictions combined, the proportion of women who were at risk for unintended pregnancy and had ongoing or potential need for contraceptive services varied significantly by age group, race/ethnicity, and urban-rural status. Among women with ongoing or potential need for contraceptive services, $15.2 \%$ used a long-acting reversible method (intrauterine device or contraceptive implant), $25.0 \%$ used a short-acting reversible method (injectable, pill, transdermal patch, or vaginal ring), and 29.5\% used a barrier or other reversible method (diaphragm, condom, withdrawal, cervical cap, sponge, spermicide, fertilityawareness-based method, or emergency contraception). In addition, $30.3 \%$ of women with ongoing or potential need were not using any method of contraception. Data in this report can be used to help guide jurisdictional planning to deliver contraceptive services, reduce unintended pregnancies, ensure that the

\footnotetext{
*Women were considered to be at risk for unintended pregnancy unless they reported 1) not being sexually active with a male partner, 2) being currently pregnant or seeking pregnancy, 3) not minding being pregnant, or 4) having had a hysterectomy.

$\dagger$ Women with ongoing or potential need for contraceptive services were defined as women considered to be at risk for unintended pregnancy who were not using permanent contraception (female sterilization or male partner vasectomy). The number of women with ongoing or potential need for contraceptive services can be used to estimate how many women might seek services.

$\$ 2017$ : Alaska, California, District of Columbia, Maine, Nevada, New Jersey, and Texas. 2019: Alabama, Arizona, Arkansas, Connecticut, Delaware, Florida, Georgia, Hawaii, Idaho, Illinois, Indiana, Iowa, Kansas, Louisiana, Maryland, Massachusetts, Minnesota, Mississippi, Missouri, Montana, Nebraska, New Mexico, New York, North Carolina, Ohio, Oklahoma, Oregon, Pennsylvania, Rhode Island, South Carolina, South Dakota, Tennessee, Utah, Virginia, West Virginia, Wisconsin, Wyoming, and Puerto Rico.
}

contraceptive needs of women and their partners are met, and evaluate efforts to increase access to contraception.

BRFSS is a state-based, random-digit-dialed telephone survey that collects self-reported health information from U.S. adults. 9 This report uses the most recent available data for the optional Family Planning Module** (2017 data for seven jurisdictions and 2019 data for 38 jurisdictions). The proportion of women aged 18-49 years at risk for unintended pregnancy was estimated. ${ }^{\dagger \dagger}$ Women were considered to be at risk for unintended pregnancy unless they reported 1) not being sexually active with a male partner, 2) being currently pregnant or seeking pregnancy, 3) not minding being pregnant, or 4) having had a hysterectomy. This approach is consistent with prior evaluation of BRFSS contraceptive use data (4). The proportion and total number of women aged 18-49 years with ongoing or potential need for contraceptive services, defined as women considered to be at risk for unintended pregnancy who were not using permanent contraception (female sterilization or male partner vasectomy), were also estimated. ${ }^{\$ \$}$ Estimates were calculated overall, by jurisdiction, and by selected sociodemographic characteristics. 99

9 https://www.cdc.gov/brfss/data_documentation/index.htm

** 2017 questions are listed in Module 17: Preconception Health/Family Health (https://www.cdc.gov/brfss/questionnaires/pdf-ques/2017_BRFSS_Pub_ Ques_508_tagged.pdf). 2019 questions are listed in Module 23: Family Planning (https://www.cdc.gov/brfss/questionnaires/pdf-ques/2019-BRFSSQuestionnaire-508.pdf).

$\dagger \dagger$ Twenty-one percent of women aged 18-49 years had missing data on being at risk for unintended pregnancy status, primarily caused by incomplete BRFSS interviews administered via mobile telephone rather than missing data specifically on the family planning module questions.

$\$ \$$ Women at risk for unintended pregnancy who did not specify the type of contraception that they used or reported "other method" (4.8\%) were excluded from estimates of women with ongoing or potential need for contraceptive services and from estimates of contraceptive use by method category. Writein responses were not available for women who responded "other method"; previous evaluation of BRFSS contraceptive use data indicates that these methods are a mix of permanent and reversible methods. https://www.cdc. gov/mmwr/volumes $/ 65 / \mathrm{wr} / \mathrm{mm} 6530 \mathrm{e} 2 . \mathrm{htm}$

99 Age was ascertained by the answer to the question, "What is your age?" Race/ ethnicity was ascertained by the answer to the question, "Which one or more of the following would you say is your race?" Health insurance coverage was ascertained by the answer to the question, "Do you have any kind of health care coverage, including health insurance, prepaid plans such as HMOs, or government plans such as Medicare, or Indian Health Service?" Routine checkup within the past year was ascertained by the answer to the question, "About how long has it been since you last visited a doctor for a routine checkup?" Urban-rural status was determined using the 2013 National Center for Health Statistics urban-rural classification scheme for counties. 
TABLE 1. Estimated numbers and percentages of women aged 18-49 years who were at risk for unintended pregnancy* and had ongoing or potential need for contraceptive services, ${ }^{\dagger}$ by jurisdiction — Behavioral Risk Factor Surveillance System, 45 jurisdictions, $2017-2019^{\S}$

\begin{tabular}{|c|c|c|c|c|}
\hline \multirow[b]{3}{*}{ Jurisdiction } & \multirow[b]{3}{*}{ Total no.? } & \multicolumn{2}{|r|}{ Women aged $18-49 \mathrm{yrs}$} & \\
\hline & & \multirow{2}{*}{$\begin{array}{l}\% \text { at risk for unintended } \\
\text { pregnancy }(95 \% \mathrm{Cl})\end{array}$} & \multicolumn{2}{|c|}{ No. and \% who had ongoing or potential need for contraceptive services } \\
\hline & & & No. $(95 \% \mathrm{Cl})^{9}$ & $\%(95 \% \mathrm{Cl})$ \\
\hline Alabama & $1,005,000$ & $76.8(73.5-79.8)$ & $571,800(533,700-610,000)$ & $56.9(53.1-60.7)$ \\
\hline Alaska & 146,300 & $67.0(60.6-72.9)$ & $75,600(65,400-85,700)$ & $51.7(44.7-58.6)$ \\
\hline Arizona & $1,444,100$ & $80.9(76.6-84.5)$ & $875,100(804,400-943,000)$ & $60.6(55.7-65.3)$ \\
\hline Arkansas & 596,100 & $75.3(70.5-79.5)$ & $347,500(316,500-377,900)$ & $58.3(53.1-63.4)$ \\
\hline California & $8,514,600$ & $70.6(67.6-73.5)$ & $5,091,700(4,810,700-5,355,700)$ & $59.8(56.5-62.9)$ \\
\hline Connecticut & 694,700 & $82.7(79.6-85.3)$ & $481,400(455,700-505,000)$ & $69.3(65.6-72.7)$ \\
\hline Delaware & 189,900 & $79.6(74.0-84.3)$ & $125,300(114,100-135,800)$ & $66.0(60.1-71.5)$ \\
\hline District of Columbia & 192,700 & $73.9(69.5-78.0)$ & $131,600(122,700-139,900)$ & $68.3(63.7-72.6)$ \\
\hline Florida & $4,130,200$ & $80.4(76.4-83.9)$ & $2,589,600(2,403,800-2,767,200)$ & $62.7(58.2-67.0)$ \\
\hline Georgia & $2,226,700$ & $84.6(81.0-87.6)$ & $1,572,100(1,474,100-1,661,100)$ & $70.6(66.2-74.6)$ \\
\hline Hawaii & 275,300 & $70.8(67.5-73.8)$ & $164,400(154,400-174,000)$ & $59.7(56.1-63.2)$ \\
\hline Idaho & 350,100 & $77.9(73.7-81.7)$ & $188,000(170,100-205,500)$ & $53.7(48.6-58.7)$ \\
\hline Illinois & $2,604,400$ & $78.6(75.1-81.7)$ & $1,669,400(1,567,800-1,763,200)$ & $64.1(60.2-67.7)$ \\
\hline Indiana & $1,349,100$ & $75.7(72.6-78.6)$ & $785,200(739,300-831,000)$ & $58.2(54.8-61.6)$ \\
\hline lowa & 613,400 & $79.4(77.0-81.6)$ & $348,400(330,600-365,600)$ & $56.8(53.9-59.6)$ \\
\hline Kansas & 573,400 & $77.6(74.9-80.0)$ & $342,900(325,700-358,900)$ & $59.8(56.8-62.6)$ \\
\hline Louisiana & 968,400 & $79.8(76.3-82.9)$ & $625,600(586,900-662,400)$ & $64.6(60.6-68.4)$ \\
\hline Maine & 244,500 & $71.5(66.8-75.8)$ & $131,300(118,800-143,800)$ & $53.7(48.6-58.8)$ \\
\hline Maryland & $1,214,500$ & $77.0(74.2-79.5)$ & $763,900(727,500-800,400)$ & $62.9(59.9-65.9)$ \\
\hline Massachusetts & $1,420,600$ & $80.1(76.9-83.0)$ & $964,600(913,400-1,012,900)$ & $67.9(64.3-71.3)$ \\
\hline Minnesota & $1,119,500$ & $78.9(76.9-80.8)$ & $690,700(665,000-715,400)$ & $61.7(59.4-63.9)$ \\
\hline Mississippi & 610,100 & $75.4(71.7-78.8)$ & $338,600(313,600-362,400)$ & $55.5(51.4-59.4)$ \\
\hline Missouri & $1,222,900$ & $78.9(75.4-82.0)$ & $694,600(644,500-743,500)$ & $56.8(52.7-60.8)$ \\
\hline Montana & 203,400 & $80.6(77.4-83.4)$ & $115,900(108,200-123,500)$ & $57.0(53.2-60.7)$ \\
\hline Nebraska & 380,800 & $80.1(76.9-83.0)$ & $243,300(229,200-256,700)$ & $63.9(60.2-67.4)$ \\
\hline Nevada & 619,000 & $73.7(68.2-78.6)$ & $359,000(322,500-394,300)$ & $58.0(52.1-63.7)$ \\
\hline New Jersey & $1,792,400$ & $74.9(71.4-78.1)$ & $1,100,500(1,032,400-1,166,900)$ & $61.4(57.6-65.1)$ \\
\hline New Mexico & 409,000 & $81.0(77.2-84.3)$ & $246,600(228,200-264,200)$ & $60.3(55.8-64.6)$ \\
\hline New York & $4,069,700$ & $81.6(77.1-85.4)$ & $2,999,400(2,800,000-3,178,400)$ & $73.7(68.8-78.1)$ \\
\hline North Carolina & $2,155,000$ & $71.3(67.1-75.2)$ & $1,204,600(1,109,800-1,299,500)$ & $55.9(51.5-60.3)$ \\
\hline Ohio & $2,281,900$ & $78.1(74.0-81.8)$ & $1,360,000(1,255,000-1,460,400)$ & $59.6(55.0-64.0)$ \\
\hline Oklahoma & 788,500 & $75.6(70.5-80.0)$ & $414,000(371,400-455,000)$ & $52.5(47.1-57.7)$ \\
\hline Oregon & 861,400 & $82.0(78.8-84.8)$ & $528,900(495,300-560,800)$ & $61.4(57.5-65.1)$ \\
\hline Pennsylvania & $2,446,600$ & $77.7(73.9-81.1)$ & $1,460,600(1,357,900-1,560,900)$ & $59.7(55.5-63.8)$ \\
\hline Rhode Island & 215,300 & $80.3(75.4-84.5)$ & $144,700(133,500-155,200)$ & $67.2(62.0-72.1)$ \\
\hline South Carolina & $1,010,300$ & $79.3(75.8-82.4)$ & $640,500(599,100-678,900)$ & $63.4(59.3-67.2)$ \\
\hline South Dakota & 170,200 & $81.4(76.4-85.5)$ & $103,100(92,900-112,700)$ & $60.6(54.6-66.2)$ \\
\hline Tennessee & $1,362,800$ & $78.4(74.2-82.0)$ & $800,000(735,900-861,300)$ & $58.7(54.0-63.2)$ \\
\hline Texas & $6,093,500$ & $67.4(63.1-71.5)$ & $3,199,100(2,918,800-3,479,400)$ & $52.5(47.9-57.1)$ \\
\hline Utah & 707,100 & $75.4(73.2-77.6)$ & $403,000(386,100-420,700)$ & $57.0(54.6-59.5)$ \\
\hline Virginia & $1,759,700$ & $78.9(75.7-81.7)$ & $1,113,900(1,050,500-1,173,700)$ & $63.3(59.7-66.7)$ \\
\hline West Virginia & 333,400 & $76.7(72.6-80.3)$ & $170,400(154,700-185,700)$ & $51.1(46.4-55.7)$ \\
\hline Wisconsin & $1,125,000$ & $79.5(74.8-83.4)$ & $717,800(661,500-770,600)$ & $63.8(58.8-68.5)$ \\
\hline Wyoming & 111,500 & $78.5(74.0-82.4)$ & $63,300(57,300-69,100)$ & $56.8(51.4-62.0)$ \\
\hline Puerto Rico & 734,000 & $75.6(72.7-78.2)$ & $332,500(309,700-356,000)$ & $45.3(42.2-48.5)$ \\
\hline Overall & $61,337,100$ & $76.2(75.4-77.0)$ & $37,231,600(36,618,200-37,783,700)$ & $60.7(59.7-61.6)$ \\
\hline
\end{tabular}

Abbreviation: $\mathrm{Cl}=$ confidence interval.

* Women were considered to be at risk for unintended pregnancy unless they reported 1) not being sexually active with a male partner, 2) being currently pregnant or seeking pregnancy, 3) not minding being pregnant, or 4) having had a hysterectomy.

+ Women with ongoing or potential need for contraceptive services were defined as women considered to be at risk for unintended pregnancy not using permanent contraception (female sterilization or male partner vasectomy). The number of women with ongoing or potential need for contraceptive services can be used to estimate how many women might seek services.

$\S$ Data shown are from 2019, except 2017 data are shown for seven jurisdictions: Alaska, California, District of Columbia, Maine, Nevada, New Jersey, and Texas.

"Weighted numbers are rounded to the nearest 100. 
TABLE 2. Estimated numbers and percentages of women aged 18-49 years who were at risk for unintended pregnancy* and had ongoing or potential need for contraceptive services, ${ }^{\dagger}$ by selected sociodemographic characteristics - Behavioral Risk Factor Surveillance System, 45 jurisdictions, 2017-2019§

\begin{tabular}{|c|c|c|c|c|}
\hline \multirow{3}{*}{$\begin{array}{l}\text { Sociodemographic } \\
\text { characteristic }\end{array}$} & \multicolumn{4}{|c|}{ Women aged $18-49$ yrs } \\
\hline & \multirow[b]{2}{*}{ Total no. 9} & \multirow{2}{*}{$\begin{array}{l}\% \text { at risk for unintended } \\
\text { pregnancy }(95 \% \mathrm{Cl})\end{array}$} & \multicolumn{2}{|c|}{ No. and \% who had ongoing or potential need for contraceptive services } \\
\hline & & & No. $(95 \% \mathrm{Cl})^{9}$ & $\%(95 \% \mathrm{Cl})$ \\
\hline \multicolumn{5}{|l|}{ Age group, yrs } \\
\hline $18-24$ & $13,992,200$ & $71.5(69.5-73.5)^{* *}$ & $9,696,600(9,402,800-9,990,400)$ & $69.3(67.2-71.4)^{* *}$ \\
\hline $25-34$ & $20,042,800$ & $74.5(73.0-75.9)$ & $12,867,500(12,546,800-13,188,200)$ & $64.2(62.6-65.8)$ \\
\hline $35-44$ & $18,901,000$ & $79.5(78.1-80.8)$ & $10,263,200(9,960,800-10,546,800)$ & $54.3(52.7-55.8)$ \\
\hline $45-49$ & $8,401,100$ & $80.4(78.6-82.1)$ & $4,435,800(4,251,000-4,620,600$ & $52.8(50.6-55.0)$ \\
\hline \multicolumn{5}{|l|}{ Race/Ethnicity } \\
\hline White, non-Hispanic & $30,888,700$ & $78.0(77.0-78.9)^{* *}$ & $18,131,700(17,791,900-18,471,400)$ & $58.7(57.6-59.8)^{* *}$ \\
\hline Black, non-Hispanic & $8,764,100$ & $75.3(73.1-77.5)$ & $5,723,000(5,503,900-5,933,300)$ & $65.3(62.8-67.7)$ \\
\hline Hispanic & $14,526,100$ & $75.5(73.6-77.2)$ & $8,948,100(8,643,000-9,238,600)$ & $61.6(59.5-63.6)$ \\
\hline Other & $6,305,400$ & $70.8(67.1-74.2)$ & $3,903,000(3,657,100-4,136,300)$ & $61.9(58.0-65.6)$ \\
\hline \multicolumn{5}{|l|}{ Insurance coverage } \\
\hline Yes & $51,112,100$ & $76.2(75.3-77.1)$ & $30,871,700(30,360,600-31,382,800)$ & $60.4(59.4-61.4)$ \\
\hline No & $9,915,300$ & $76.8(74.7-78.8)$ & $6,167,300(5,929,300-6,395,400)$ & $62.2(59.8-64.5)$ \\
\hline \multicolumn{5}{|c|}{ Routine checkup within past yr } \\
\hline Yes & $43,835,000$ & $76.7(75.7-77.6)$ & $26,651,700(26,213,300-27,133,900)$ & $60.8(59.8-61.9)$ \\
\hline No & $16,704,300$ & $75.3(73.6-76.9)$ & $10,056,000(9,755,300-10,356,700)$ & $60.2(58.4-62.0)$ \\
\hline \multicolumn{5}{|l|}{ Urban-rural status $^{\dagger \dagger}$} \\
\hline Urban & $57,369,500$ & $76.5(75.6-77.3)^{* *}$ & $35,282,200(34,708,500-35,798,600)$ & $61.5(60.5-62.4)^{* *}$ \\
\hline Rural & $3,227,300$ & $72.6(69.6-75.4)$ & $1,639,500(1,552,300-1,729,800)$ & $50.8(48.1-53.6)$ \\
\hline
\end{tabular}

Abbreviation: $\mathrm{Cl}=$ confidence interval.

* Women were considered to be at risk for unintended pregnancy unless they reported 1) not being sexually active with a male partner, 2) being currently pregnant or seeking pregnancy, 3) not minding being pregnant, or 4) having had had a hysterectomy.

† Women with ongoing or potential need for contraceptive services were defined as women considered to be at risk for unintended pregnancy not using permanent contraception (female sterilization or male partner vasectomy). The number of women with ongoing or potential need for contraceptive services can be used to estimate how many women might seek services.

$\S$ 2017: Alaska, California, District of Columbia, Maine, Nevada, New Jersey, and Texas. 2019: Alabama, Arizona, Arkansas, Connecticut, Delaware, Florida, Georgia, Hawaii, Idaho, Illinois, Indiana, lowa, Kansas, Louisiana, Maryland, Massachusetts, Minnesota, Mississippi, Missouri, Montana, Nebraska, New Mexico, New York, North Carolina, Ohio, Oklahoma, Oregon, Pennsylvania, Rhode Island, South Carolina, South Dakota, Tennessee, Utah, Virginia, West Virginia, Wisconsin, Wyoming, and Puerto Rico.

I Weighted numbers are rounded to the nearest 100.

** $p<0.05$ for chi-square test comparing the distribution of the outcome by the sociodemographic characteristic.

${ }^{+t}$ Determined using the 2013 National Center for Health Statistics urban-rural classification scheme for counties. https://www.cdc.gov/nchs/data_access/urban_rural.htm

Chi-square tests were conducted to examine differences in distributions by sociodemographic characteristics.

Among women with ongoing or potential need for contraceptive services, the proportions who were using a specific contraceptive method at last sexual encounter were estimated ${ }^{* * *}$ by category of method or no method, overall, and by jurisdiction. Categories of contraceptive methods reflect different levels of effort for method initiation and continuation. Long-acting reversible contraception methods include intrauterine devices and contraceptive implants; these methods require the most clinical effort for initiation but require minimal follow-up until time for removal or reinsertion and minimal action by the woman. Short-acting reversible contraception methods include injectables, pills, transdermal patches, and vaginal

\footnotetext{
*** Contraceptive method use at last sexual encounter was ascertained by the answers to the questions, "The last time you had sex with a man, did you or your partner do anything to keep you from getting pregnant?" (2019) or "What did you or your partner do the last time you had sex to keep you from getting pregnant?" (2017).
}

rings; these methods require less clinical effort for initiation than long-acting reversible methods but require ongoing clinical services and supplies and action by the woman to maintain use. Barrier or other reversible methods included diaphragms, condoms (male or female), withdrawal, cervical caps, sponges, spermicides, fertility-awareness-based methods, and emergency contraception; these methods have little or no need for clinical services for initiation but require action by the woman or her partner to maintain use. Analyses were conducted using SAS (version 9.4; SAS Institute) with SUDAAN to account for the complex sampling methods used in BRFSS; data were weighted for nonresponse. ${ }^{\dagger \dagger}$

Among women aged 18-49 years in the 45 jurisdictions, $76.2 \%$ were considered to be at risk for unintended pregnancy,

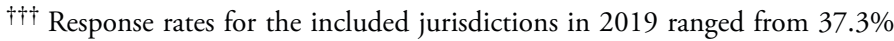
(New York) to 73.1\% (South Dakota) (https://www.cdc.gov/brfss/annual_ data/2019/pdf/2019-response-rates-table-508.pdf). Response rates for the included jurisdictions in 2017 ranged from $31.4 \%$ (California) to $49.3 \%$ (Maine). https://www.cdc.gov/brfss/annual_data/2017/pdf/2017-responserates-table-508.pdf 
TABLE 3. Percentages of women aged 18-49 years who had ongoing or potential need for contraceptive services* using specific contraceptive methods, by category of method ${ }^{\dagger}$ or no method and jurisdiction - Behavioral Risk Factor Surveillance System, 45 jurisdictions, 2017-2019§

$\%$ of women aged $18-49$ yrs $(95 \% \mathrm{Cl})$

\begin{tabular}{|c|c|c|c|c|}
\hline \multirow[b]{2}{*}{ Jurisdiction } & \\
\hline & $\begin{array}{l}\text { Long-acting } \\
\text { reversible method }\end{array}$ & $\begin{array}{l}\text { Short-acting } \\
\text { reversible method }\end{array}$ & $\begin{array}{l}\text { Barrier or other } \\
\text { reversible method }\end{array}$ & No method \\
\hline Alabama & $9.6(7.1-12.9)$ & $25.1(20.7-30.1)$ & $37.0(31.9-42.3)$ & $28.3(23.7-33.3)$ \\
\hline Alaska & 28.5 (19.6-39.5) & $18.6(11.9-27.8)$ & $31.6(21.9-43.1)$ & $21.3(13.9-31.4)$ \\
\hline Arizona & $13.0(9.5-17.5)$ & 24.5 (19.3-30.6) & $28.3(22.8-34.4)$ & $34.3(28.5-40.5)$ \\
\hline Arkansas & $9.8(6.2-15.2)$ & $25.2(19.4-32.0)$ & $30.1(23.6-37.5)$ & $35.0(28.6-41.9)$ \\
\hline California & $15.1(12.5-18.2)$ & $26.9(23.1-31.0)$ & $30.4(26.6-34.4)$ & $27.6(23.9-31.6)$ \\
\hline Connecticut & $16.1(12.5-20.4)$ & $23.8(19.9-28.2)$ & $31.1(26.7-35.9)$ & $29.0(24.8-33.6)$ \\
\hline Delaware & $12.5(8.8-17.6)$ & $28.1(21.8-35.4)$ & 30.1 (23.6-37.6) & $29.2(22.9-36.5)$ \\
\hline District of Columbia & $11.5(8.1-15.9)$ & $16.0(12.3-20.7)$ & $42.6(37.1-48.3)$ & $29.9(25.2-35.0)$ \\
\hline Florida & $12.9(9.8-16.8)$ & $25.8(21.2-31.0)$ & $28.9(23.9-34.4)$ & $32.5(27.4-38.0)$ \\
\hline Georgia & $11.2(8.2-15.0)$ & $25.3(20.3-31.1)$ & $26.2(21.3-31.9)$ & $37.3(31.6-43.3)$ \\
\hline Hawaii & $16.9(13.6-20.7)$ & $21.4(18.0-25.3)$ & $22.3(18.9-26.1)$ & $39.4(34.8-44.2)$ \\
\hline Idaho & $29.4(23.5-36.1)$ & $22.4(17.0-28.9)$ & $24.0(18.4-30.6)$ & $24.2(19.1-30.2)$ \\
\hline Illinois & $10.4(8.1-13.2)$ & $23.8(20.0-28.1)$ & $35.4(30.9-40.2)$ & $30.4(26.0-35.2)$ \\
\hline Indiana & $14.0(11.2-17.4)$ & $24.7(20.8-29.2)$ & $29.7(25.6-34.2)$ & 31.5 (27.4-35.9) \\
\hline lowa & $22.3(19.3-25.7)$ & $28.9(25.5-32.7)$ & $25.2(22.0-28.8)$ & $23.5(20.5-26.8)$ \\
\hline Kansas & $19.2(16.3-22.4)$ & $27.7(24.3-31.4)$ & 23.7 (20.3-27.6) & $29.4(26.0-33.1)$ \\
\hline Louisiana & $11.1(8.1-14.9)$ & $25.2(20.9-30.0)$ & 34.3 (29.4-39.6) & $29.4(25.2-34.0)$ \\
\hline Maine & $33.6(26.8-41.2)$ & $26.7(20.6-33.8)$ & $19.3(14.3-25.5)$ & $20.4(15.7-26.2)$ \\
\hline Maryland & $16.1(13.5-19.1)$ & $24.4(21.1-28.1)$ & $33.4(29.8-37.2)$ & $26.1(22.9-29.6)$ \\
\hline Massachusetts & $19.5(16.3-23.2)$ & $28.6(24.5-33.0)$ & $31.8(27.6-36.2)$ & $20.1(16.6-24.1)$ \\
\hline Minnesota & 21.9 (19.6-24.4) & $28.5(25.8-31.3)$ & $23.5(21.0-26.1)$ & $26.2(23.6-28.9)$ \\
\hline Mississippi & $10.3(7.4-14.1)$ & $24.7(20.1-29.9)$ & $34.1(29.0-39.7)$ & $30.9(26.2-36.1)$ \\
\hline Missouri & $16.5(12.8-21.1)$ & $27.7(22.8-33.1)$ & $31.8(26.6-37.4)$ & $24.1(19.5-29.3)$ \\
\hline Montana & $29.2(24.8-34.1)$ & $23.2(19.3-27.6)$ & 24.0 (19.9-28.6) & $23.6(19.8-27.9)$ \\
\hline Nebraska & $17.1(13.5-21.5)$ & $27.0(22.7-31.7)$ & $28.7(24.5-33.4)$ & $27.1(23.2-31.5)$ \\
\hline Nevada & $21.2(15.5-28.3)$ & $22.7(16.9-29.8)$ & $23.6(17.3-31.3)$ & $32.5(25.6-40.1)$ \\
\hline New Jersey & $12.6(9.8-16.0)$ & $22.7(18.6-27.4)$ & $31.1(26.7-35.8)$ & $33.6(29.0-38.6)$ \\
\hline New Mexico & $22.5(18.0-27.7)$ & $18.0(13.8-23.1)$ & $27.7(22.7-33.4)$ & $31.8(26.5-37.6)$ \\
\hline New York & $14.1(10.2-19.2)$ & $18.1(14.1-22.9)$ & $33.7(28.0-39.9)$ & $34.1(28.4-40.2)$ \\
\hline North Carolina & $20.0(15.6-25.1)$ & $24.4(19.8-29.6)$ & 23.7 (19.2-28.9) & $32.0(26.8-37.6)$ \\
\hline Ohio & $15.9(11.7-21.3)$ & $30.8(25.4-36.9)$ & 23.5 (18.8-29.0) & $29.8(24.5-35.6)$ \\
\hline Oklahoma & 16.5 (11.9-22.6) & $29.7(23.1-37.1)$ & $26.7(20.6-33.8)$ & $27.1(21.4-33.7)$ \\
\hline Oregon & $30.7(26.5-35.3)$ & $24.2(20.5-28.4)$ & $24.8(21.1-29.0)$ & $20.2(16.5-24.5)$ \\
\hline Pennsylvania & $13.9(11.0-17.3)$ & $30.6(25.9-35.8)$ & $29.4(24.5-34.9)$ & $26.1(21.6-31.1)$ \\
\hline Rhode Island & $16.6(12.3-22.1)$ & $31.0(25.3-37.4)$ & $26.6(21.0-33.0)$ & $25.8(20.7-31.7)$ \\
\hline South Carolina & $11.9(9.1-15.4)$ & $28.4(24.0-33.3)$ & $30.8(26.2-35.8)$ & $28.9(24.2-34.1)$ \\
\hline South Dakota & $17.6(12.7-23.9)$ & $27.7(21.2-35.2)$ & $26.7(20.0-34.7)$ & $27.9(21.4-35.7)$ \\
\hline Tennessee & 10.5 (7.4-14.7) & 32.9 (27.3- 38.9) & $28.2(22.6-34.6)$ & $28.4(23.2-34.2)$ \\
\hline Texas & $12.0(8.6-6.5)$ & $18.3(14.3-23.3)$ & $30.3(24.7-36.7)$ & $39.3(33.2-45.8)$ \\
\hline Utah & 36.1 (33.0-39.3) & $21.9(19.2-24.8)$ & $21.2(18.7-24.0)$ & $20.8(18.3-23.6)$ \\
\hline Virginia & $17.4(14.3-21.0)$ & $27.3(23.1-31.9)$ & $25.1(21.5-29.0)$ & $30.3(26.0-34.9)$ \\
\hline West Virginia & $16.6(12.2-22.3)$ & $25.9(20.4-32.2)$ & $27.1(20.9-34.3)$ & $30.4(24.7-36.8)$ \\
\hline Wisconsin & $18.4(13.8-24.0)$ & $32.4(26.8-38.4)$ & $25.7(20.5-31.6)$ & $23.6(18.8-29.1)$ \\
\hline Wyoming & $18.7(13.6-25.1)$ & $23.3(17.5-30.3)$ & $27.9(21.6-35.3)$ & $30.1(23.4-37.8)$ \\
\hline Puerto Rico & $6.9(4.7-10.0)$ & $13.7(10.8-17.4)$ & $33.5(29.1-38.2)$ & $45.8(41.1-50.7)$ \\
\hline Overall & $15.2(14.4-16.0)$ & $25.0(24.0-26.0)$ & $29.5(28.4-30.6)$ & $30.3(29.2-31.5)$ \\
\hline
\end{tabular}

Abbreviation: $\mathrm{Cl}=$ confidence interval.

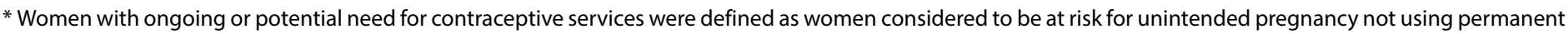

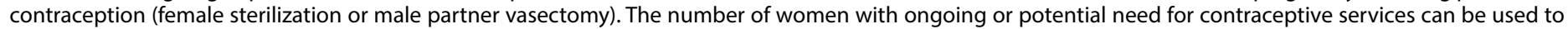
estimate how many women might seek services.

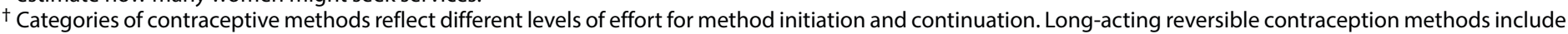

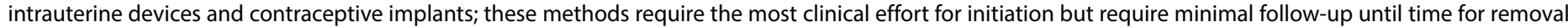

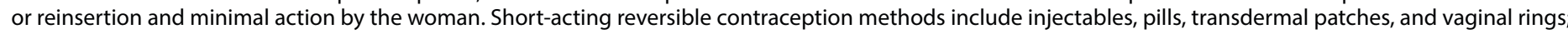

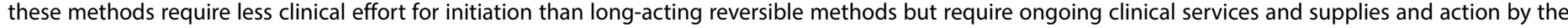

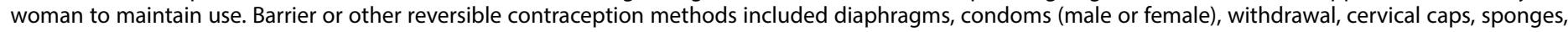

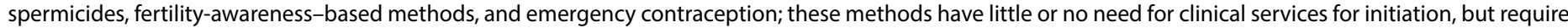
action by the woman or her partner to maintain use.

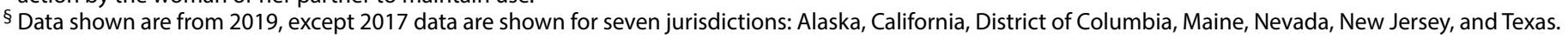




\section{Summary}

What is already known about this topic?

Ensuring access to contraception is important for promoting reproductive autonomy, preventing unintended pregnancies, and promoting optimal and equitable reproductive health.

What is added by this report?

Analysis of 2017-2019 Behavioral Risk Factor Surveillance System data from 45 jurisdictions found that $60.7 \%$ of women aged 18-49 years had ongoing or potential need for contraceptive services; estimates varied by jurisdiction, age group, race/ ethnicity, and urban-rural status. Nearly one third (30.3\%) of women with ongoing or potential need were not using any method of contraception at last sexual encounter.

What are the implications for public health practice?

Jurisdictions can use the data provided in this report to plan delivery of contraceptive services and evaluate efforts to increase access to contraception.

ranging from $67.0 \%$ (Alaska) to $84.6 \%$ (Georgia); $60.7 \%$ had ongoing or potential need for contraceptive services, ranging from $45.3 \%$ (Puerto Rico) to $73.7 \%$ (New York) (Table 1). For all jurisdictions combined, the proportion of women who were at risk for unintended pregnancy and had need for contraceptive services varied significantly by age group, race/ethnicity, and urban-rural status (Table 2). Although these proportions did not vary by insurance coverage or routine checkup within the past year, among women with ongoing or potential need for contraceptive services, $16.7 \%$ did not have insurance, and $27.4 \%$ did not have a routine checkup within the past year.

Among women with ongoing or potential need for contraceptive services, $15.2 \%$ used a long-acting reversible method, $25.0 \%$ used a short-acting reversible method, $29.5 \%$ used a barrier or other reversible method, and $30.3 \%$ used no method (Table 3). By jurisdiction, among women with ongoing or potential need for contraceptive services, use of a long-acting reversible method ranged from $6.9 \%$ (Puerto Rico) to 36.1\% (Utah), use of a short-acting reversible method ranged from $13.7 \%$ (Puerto Rico) to $32.9 \%$ (Tennessee), use of a barrier or other reversible method ranged from $19.3 \%$ (Maine) to $42.6 \%$ (District of Columbia), and use of no method ranged from 20.1\% (Massachusetts) to 45.8\% (Puerto Rico).

\section{Discussion}

During 2017-2019, six out of 10 (60.7\%, approximately 37.2 million women) women aged 18-49 years in the 45 jurisdictions had ongoing or potential need for contraceptive services, with variation observed by jurisdiction, age group, race/ethnicity, and urban-rural status. An estimated 6.2 million women without insurance had ongoing or potential need for contraceptive services and might require publicly funded care. Among women with ongoing or potential need for contraceptive services, nearly one in three (30.3\%) was not using contraception at last sexual encounter. Improving contraception access and uptake among these women might have a large effect on meeting reproductive health care needs and reducing unintended pregnancies (5). In addition, nearly one in three women $(29.5 \%)$ used a barrier or other reversible method (i.e., diaphragm, condom, withdrawal, cervical cap, sponge, spermicide, fertility-awareness-based method, or emergency contraception); given lower effectiveness of these methods for preventing unintended pregnancy during typical use compared with long-acting and short-acting reversible methods (G), jurisdictions might consider efforts to ensure women's access to the full range of available contraceptive methods. These efforts might include removing logistic and administrative barriers for contraceptive services and supplies, training providers, increasing provider reimbursement, and increasing consumer awareness of available contraceptive services and method options ( 7 ).

Jurisdiction-level information on unintended pregnancy risk and need for contraceptive services is important because unintended pregnancy rates vary by jurisdiction (8). Variation might be caused by differences in population characteristics (9) or differences in the implementation of public health programs and policies to increase contraceptive use. Examples of programs and policies that vary by jurisdiction include participation in a learning community to increase contraception access

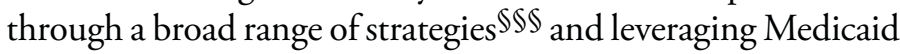
coverage to expand eligibility for family planning services.999 Jurisdiction-level information is also important for planning, enhancing, and evaluating efforts to improve contraception access and services. Contraceptive care clinical performance measures use administrative data to assess health system quality of care (10), but these data lack information on unintended pregnancy risk. Data in this report can be used by jurisdictions to calculate the measures ${ }^{* * * *}$ to better reflect their populations in need. The data provided in this report also have implications for ensuring access to contraceptive services during public health emergencies that have a disproportionate impact on

\footnotetext{
$\$ \mathbb{S}$ The learning community was convened by the Association of State and Territorial Health Officials in collaboration with CDC, Centers for Medicare \& Medicaid Services, and the U.S. Department of Health and Human Services Office of Population Affairs. Examples of strategies to improve contraceptive use included improving provider awareness and training; addressing logistical, stocking, and administrative barriers; improving reimbursement and financial sustainability; and ensuring adequate service locations. https://www.astho.org/Programs/Maternal-and-Child-Health/ Increasing-Access-to-Contraception/

999 ht ps://www.guttmacher.org/state-policy/explore/ medicaid-family-planning-eligibility-expansions

**** https://opa.hhs.gov/research-evaluation/title-x-services-research/ contraceptive-care-measures/most-or-moderately
} 
reproductive-aged women or on the delivery of routine clinical services. BRFSS data were previously used to estimate the number of women needing contraceptive services during the 2016 Zika virus outbreak because of concerns about Zika virus-related adverse pregnancy and birth outcomes (4). In emergencies that disrupt routine care, jurisdictions can use data on the number of women needing contraceptive services and current contraceptive use to help plan for potential alternative models for service provision (e.g., telehealth/telemedicine) and for ensuring continued access to method supply (e.g., providing or prescribing a 1-year supply of methods that need resupply). ${ }^{\dagger \dagger \dagger \dagger}$

The findings provided in this report are subject to at least four limitations. First, information on contraceptive use was self-reported and might be subject to recall error or social desirability bias. Second, for $21 \%$ of women aged $18-49$ years, data on the family planning module were missing, mostly a result of incomplete BRFSS interviews. Although the proportion of women for whom these data were missing was generally similar by jurisdiction, a higher proportion of younger women and women who self-identified as non-Hispanic Black or other had missing data. Third, findings apply only to the jurisdictions that implemented the optional family planning module and therefore might not be generalizable to the entire U.S. population of women aged 18-49 years. Finally, nonresponse bias remains a possibility, although the BRFSS weighting methodology adjusts for non response.

Ensuring access to contraceptive services is important to promote reproductive autonomy, prevent unintended pregnancies, and promote optimal and equitable reproductive health. Understanding the need for contraceptive services, including the number of uninsured women who might need publicly supported care, can help jurisdictions plan health care delivery to support women and their partners in choosing whether and when to become pregnant. The data in this report can be used by jurisdictions to estimate the number of women who might seek contraceptive services and to plan and evaluate contraception access policies and programs.

$\overline{\dagger \dagger \dagger}$ https://rhntc.org/resources/what-family-planning-providers-can-do-meetclient-needs-during-covid-19?utm_source $=$ eNews\&utm_campaign=March
Corresponding author: Lauren B. Zapata, lzapata@cdc.gov, 770-488-6358.

${ }^{1}$ Division of Reproductive Health, National Center for Chronic Disease
Prevention and Health Promotion, CDC; ${ }^{2}$ Division of Human Development
and Disability, National Center on Birth Defects and Developmental
Disabilities, CDC; ${ }^{3}$ Iowa Department of Public Health; ${ }^{4}$ Immunization Services
Division, National Center for Immunization and Respiratory Diseases, CDC.

All authors have completed and submitted the International Committee of Medical Journal Editors form for disclosure of potential conflicts of interest. No potential conflicts of interest were disclosed.

\section{References}

1. Finer LB, Zolna MR. Declines in unintended pregnancy in the United States, 2008-2011. N Engl J Med 2016;374:843-52. PMID:26962904 https://doi.org/10.1056/NEJMsa1506575

2. Gipson JD, Koenig MA, Hindin MJ. The effects of unintended pregnancy on infant, child, and parental health: a review of the literature. Stud Fam Plann 2008;39:18-38. PMID:18540521 https://doi. org/10.1111/j.1728-4465.2008.00148.x

3. Holt K, Reed R, Crear-Perry J, Scott C, Wulf S, Dehlendorf C. Beyond same-day long-acting reversible contraceptive access: a person-centered framework for advancing high-quality, equitable contraceptive care. Am J Obstet Gynecol 2020;222(4S):S878.e1-6. PMID:31809706 https:// doi.org/10.1016/j.ajog.2019.11.1279

4. Pazol K, Ellington SR, Fulton AC, et al.; Behavioral Risk Factor Surveillance System Family Planning Module Working Group. Contraceptive use among women at risk for unintended pregnancy in the context of public health emergencies-United States, 2016. MMWR Morb Mortal Wkly Rep 2018;67:898-902. PMID:30114001 https:// doi.org/10.15585/mmwr.mm6732a6

5. Thomas A, Karpilow Q. The intensive and extensive margins of contraceptive use: comparing the effects of method choice and method initiation. Contraception 2016;94:160-7. PMID:27036300 https:// doi.org/10.1016/j.contraception.2016.03.014

6. Trussell J, Aiken ARA, Micks E, Guthrie KA. Efficacy, safety, and personal considerations. In: Hatcher RA, Nelson AL, Trussell J, et al, eds. Contraceptive technology. 21st ed. New York, NY: Ayer Company Publishers, Inc.; 2018.

7. Kroelinger CD, Romero L, Lathrop E, et al. Meeting summary: state and local implementation strategies for increasing access to contraception during Zika preparedness and response-United States, September 2016. MMWR Morb Mortal Wkly Rep 2017;66:1230-5. PMID:29121000 https://doi.org/10.15585/mmwr.mm6644a6

8. Finer LB, Kost K. Unintended pregnancy rates at the state level. Perspect Sex Reprod Health 2011;43:78-87. PMID:21651706 https://doi. org/10.1363/4307811

9. Janis JA, Ahrens KA, Kozhimannil KB, Ziller EC. Contraceptive method use by rural-urban residence among women and men in the United States, 2006 to 2017. Women's Health Issues 2021;31:277-85. PMID:33531190 https://doi.org/10.1016/j.whi.2020.12.009

10. Gavin L, Frederiksen B, Robbins C, Pazol K, Moskosky S. New clinical performance measures for contraceptive care: their importance to healthcare quality. Contraception 2017;96:149-57. PMID:28596123 https://doi.org/10.1016/j.contraception.2017.05.013 